\title{
The Simulation of band-notched UWB antennas Based on CST
}

\author{
Ke Zhang, Tian-Peng Xia, Jinghang Zou*, Gao-Chong Lv and Wei-Wei Wen \\ College of Information Engineering, Beijing Institute of Petrochemical Technology, \\ Beijing, China, 102600 \\ E-mail: zhangke@bipt.edu.cn \\ *Corresponding author
}

\begin{abstract}
The Ultra Wide band (UWB) antennas play an important role in UWB system. As a crucial component of the UWB system, the UWB antennas affect performance of the system significantly and have advantages of low profile, low power consumption and integration easily, so they are widely used in communication systems. The Federal Communication Commission (FCC) authorized the unlicensed use of the UWB $(3.1 \mathrm{GHz} \sim 10.6 \mathrm{GHz})$ in February 2002. This dissertation firstly gives a brief introduction on the development history and research status of UWB antenna. Secondly, the main performance parameters, the method and the performance of the UWB antenna are analyzed in detail. Combined with these characteristics of UWB system, this paper provides the design requirements of ultra-wide band antenna. Then, based on the above research and analysis, the author designs an ultra-wide band antenna structure by using CST MWS simulation software, and realizes the WLAN band notched performance by loading structure. Finally the author comes to the conclusion.
\end{abstract}

Keywords: Ultra Wide Band; UWB Antenna; Band-notched Antenna

\section{Introduction}

Ultra wide band technology has been developed for more than 50 years, which using an ultra short pulse with very wide spectrum to communicate. It is also known for base band communication, and is wide used in radar, sensing and military communications systems.

Antenna plays an important role in wireless communication system, and its function is to radiate or receive electromagnetic wave in space. As the front of the receiving signal, There are many requirements for the UWB antenna. For example, if Ultra wide band was guaranteed, antenna structure also need to be compact to integrate conveniently. And how to avoid mutual interference with other antennas due to operating frequency bandwidth[1]. Therefore, the design of ultra wide band antenna is facing a greater challenge. 


\section{UWB Antenna Characteristic Parameters}

\subsection{Antenna Input Impedance}

The input impedance of the antenna is a parameter that reflects the characteristic of the antenna circuit. The impedance of the antenna input impedance is the input impedance of the antenna:

$$
\boldsymbol{Z}_{\text {in }}=\frac{\boldsymbol{V}_{\text {in }}}{\boldsymbol{I}_{\text {in }}}=2 \frac{\boldsymbol{P}_{\text {in }}}{\left|\boldsymbol{I}_{\text {in }}\right|^{2}}=\boldsymbol{R}_{\text {in }}+\boldsymbol{j} \boldsymbol{X}_{\text {in }}
$$

Among them, $\boldsymbol{P}_{\boldsymbol{i n}}$ is the input power of the antenna, $\boldsymbol{V}_{\boldsymbol{i n}}$ is the input voltage of the antenna, $\boldsymbol{I}_{\text {in }}$ is the input current of the antenna, $\boldsymbol{R}_{\boldsymbol{i n}}$ is the input resistance of the antenna, including the input current loss resistance $\boldsymbol{R}_{\boldsymbol{l}}$ and the radiation resistance $\boldsymbol{R}_{\text {rad }} . \boldsymbol{X}_{\text {in }}$ is the input reactance of the antenna, which converts a part of the input power into energy, stored in the vicinity of the antenna surface, rather than to the free space.

\subsection{Impedance Bandwidth And Reflection Coefficient}

Impedance bandwidth is the bandwidth of less than $10 \%$ when the impedance of the antenna and the transmission line is matched. Impedance bandwidth has two main characteristic parameters: Voltage standing wave ratio (VSWR)and return $\operatorname{loss}(\mathrm{RL})$.These two parameters relate to the reflection coefficient of terminal voltage $\Gamma$.A transmission line model as shown in figure $1 . \Gamma$ is defined as the ratio between the reflection wave and incident wave voltage. From theoretical knowledge of transmission lines can get its expression:

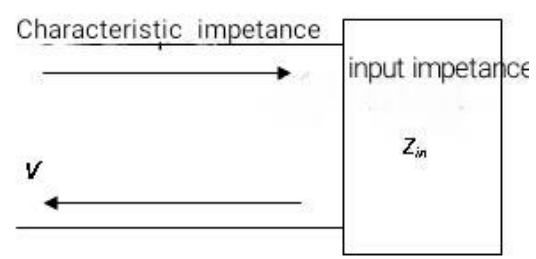

Fig. 1 Transmission line model

$$
\Gamma=\frac{V^{-}}{V^{+}}=\frac{Z_{c}-Z_{i n}}{Z_{c}+Z_{i n}}
$$

The incident power is:

$$
\boldsymbol{P}^{+}=\frac{\left|\boldsymbol{V}^{+}\right|^{2}}{2 \boldsymbol{Z}_{c}}
$$

Reflected power is: 


$$
\boldsymbol{P}^{-}=|\boldsymbol{\Gamma}|^{2} \frac{\left|\boldsymbol{V}^{+}\right|^{2}}{2 \boldsymbol{Z}_{c}}
$$

The final power of the antenna is:

$$
\boldsymbol{P}_{\text {in }}=\left(1-|\boldsymbol{\Gamma}|^{2}\right) \frac{\left|\boldsymbol{V}^{+}\right|^{2}}{2 \boldsymbol{Z}_{\boldsymbol{C}}}
$$

In the working band, the reflected power of the antenna should be less than $10 \%$ of the incident power, By formula (5) can be solved, the reflection coefficient $\Gamma$ is less than $0.3162[3]$.

\section{UWB Antenna Design}

\subsection{Requirements of antenna design}

(1) The working band covering the UWB $(3.1-10.6 \mathrm{GHz})$;

(2) In the working band, VSWR is less than 2;

(3) The structure is simple, convenient to make and easy to load the structure;

\subsection{Antenna Initial Model}

The structure of monopole UWB antenna is shown in Fig. (2). The whole structure is composed of the radiating element, the floor and the coaxial feeder. In the same axis, the conductor through the floor connect to the radiating element, and the outer conductor is grounded.

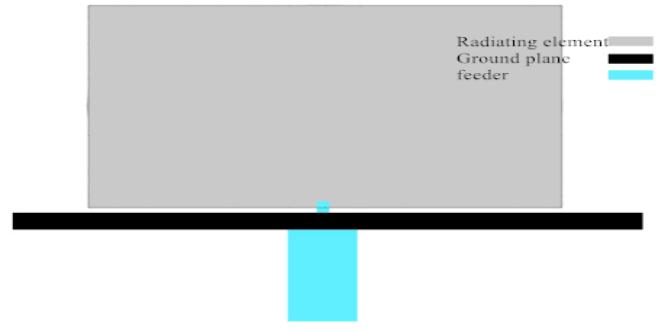

Fig. 2 Schematic diagram of rectangular antenna

Using CST software to simulate the antenna, and the return loss S11 shown in Fig. 3. From the graph, we can see that the antenna cannot work in UWB. nd the $\mathrm{S} 11$ in the band were more than $-10 \mathrm{~dB}$, so the antenna cannot meet the requirement of the antenna design. The main work of this article is to increase the impedance bandwidth of the antenna by optimizing the antenna size on the promise of not increase the size of the antenna [4]. 


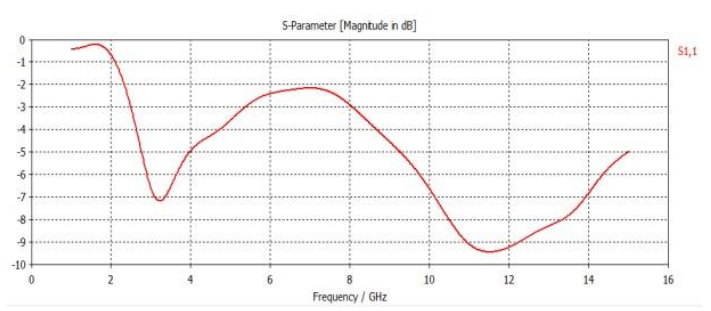

Fig. 3 The return loss of the rectangular antenna

\subsection{The radiator adopts elliptic structure}

From Antenna surface map Fig. (4) ,it is easy to get antenna surface current distribution at frequency point $(5.3 \mathrm{GHz})$.Feeding port generated a large amount of current and these currents form standing waves or reflected back, so that the emission energy has been a lot of loss, can't be radiated out. The reason for the increase of reflection loss may due to the abrupt change of the radiation plane in the rectangle.

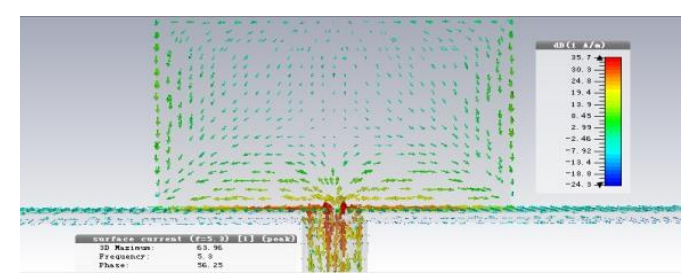

Fig. 4 The surface current of rectangular structure

Under the premise of not increasing the size of the antenna, this paper makes a good match between the whole antenna and the feed network by using the elliptical gradient structure, thus the impedance bandwidth of the antenna is extended. To maintain the basic size of the antenna unchanged, to change the rectangle for the oval structure [12], than the antenna schematic diagram shown in Fig. 5:

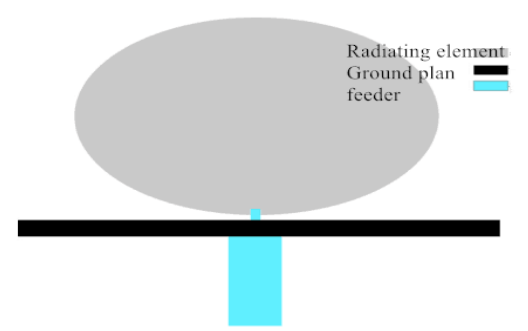

Fig. 5 Schematic diagram of elliptical structure 
After simulation, the return loss of the elliptical antenna is shown in Figure6. Visible, when $\mathrm{S} 11$ is less than - $10 \mathrm{~dB}$, the bandwidth of the antenna is 3.1$15 \mathrm{GHz}$, covering the range of UWB frequency. Thus it may be known, the rectangular plane radiation instead of gradient edge oval. The impedance bandwidth of the antenna is obviously improved, witch satisfy the design requirements.

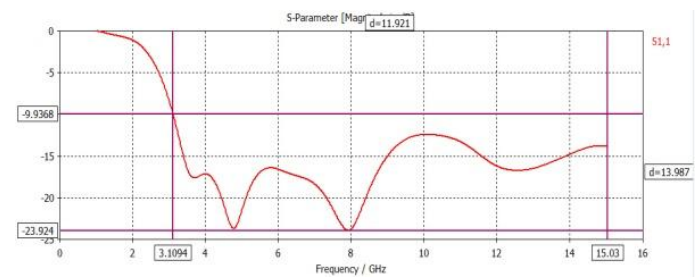

Fig. 6 The return loss of elliptical antenna

\subsection{Antenna Optimization}

On the oval semi minor axis of the selected point and to the point as the center of the circle, the point to the elliptic boundary radius, dig a circular groove, so that in the slot loading parasitic structure without affecting the antenna size, the optimization design of elliptical antenna schematic diagram as shown in figure 7 shows. Figure 8 for the improved circular groove antenna with different radius of influence on echo reflection S11 Visible, in the appropriate range of choice of different radius of $\mathrm{S} 11$ has little impact on the, and are in line with the requirements of ultra wide band.[5]
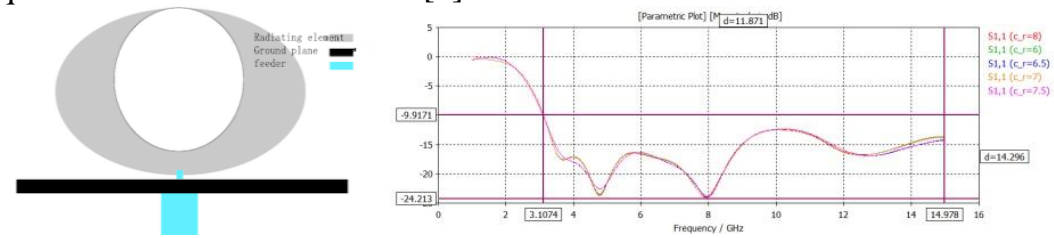

Fig. 7 Schematic diagram of the optimized antenna Fig. 8 The parameters of circular radius

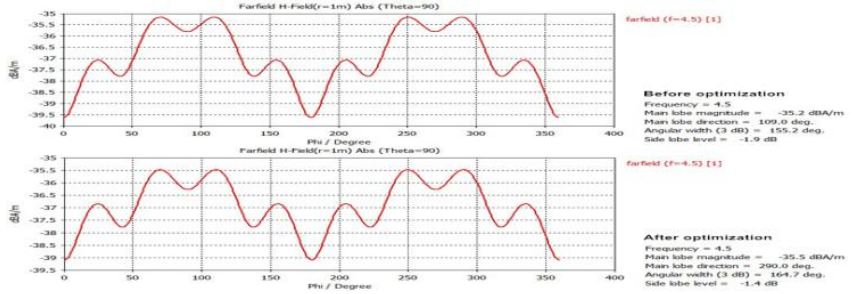

Fig. 9 Comparison of $\mathrm{H}$ surface orientation before and after optimization

Figure 9 for the rectangular coordinate system under the $\mathrm{H}$ surface direction, visible, structural optimization of the antenna $\mathrm{H}$ surface radiation intensity than before the optimization increased by $0.3 \mathrm{~dB}$. 


\subsection{Ultra wide band antenna measurement}

Through software simulation analysis, the UWB antenna scheme is feasible. Figure 10 for the UWB antenna practical model, the use of vector network analyzer for the measurement of the antenna, as shown in figure 11.

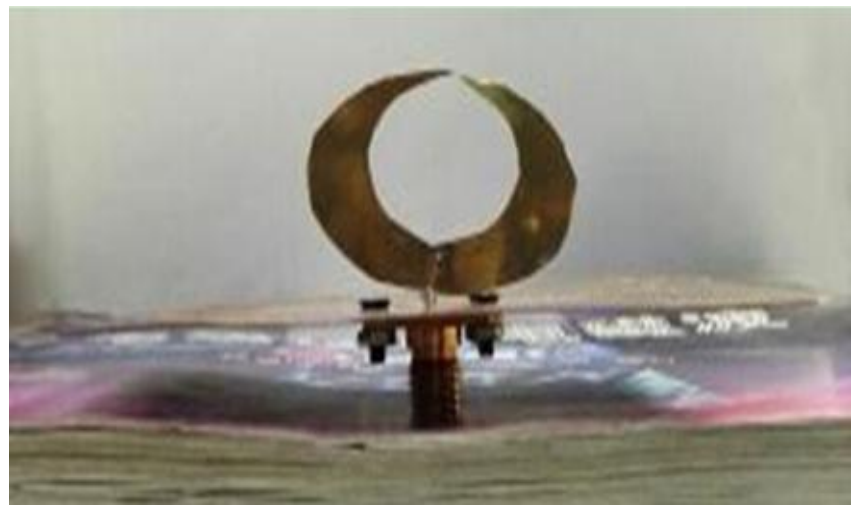

Fig. 10 The practical model of ultra wide band antenna

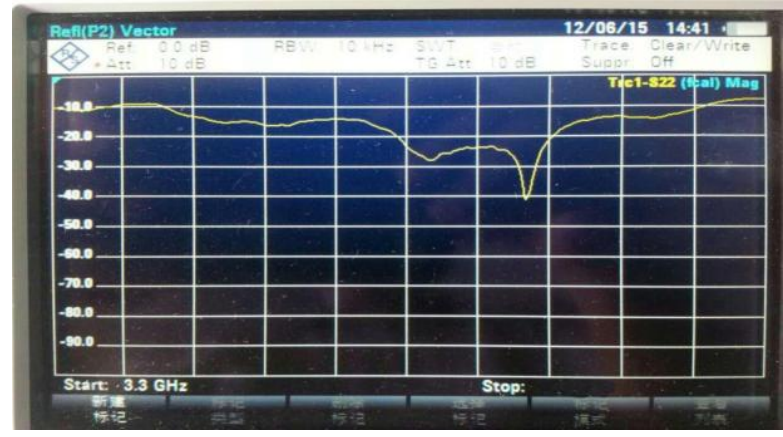

Fig. 11 The measured S11 of ultra wide band antenna

Comparison figure 11 and figure 8 can be seen that there is a certain difference between the simulation results and the measured results, but the two S11 curves are basically the same, and the measured results are in line with the requirements of UWB bandwidth.

\section{UWB Antenna With Notch Function}

\subsection{Notch Characteristic Realization}

The size and shape of the parasitic structure are very important to the performance of the antenna. This design first will open structure for T type, then in order to achieve in the WLAN band depression belt and open structure is 
changed into a cross shaped, final adjustment of antenna parameters, improve the antenna properties, it has the design requirements of the performance [6].

Fig. 12 shows a schematic diagram of an ultra wide band antenna with notch characteristics. The antenna is improved on the basis of the design of the ultra wide band antenna in the last chapter. At the bottom of the circular groove of the upper part of the antenna, a T type or cross type structure is added, and the corresponding structure size is adjusted to achieve the purpose of trap wave.

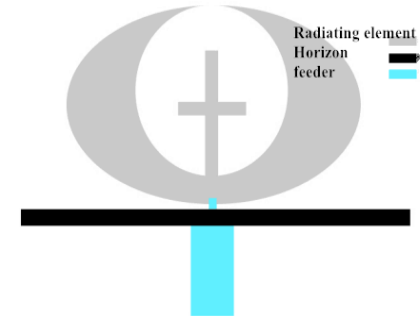

Fig. 12 Schematic diagram of an ultra wide band antenna with notch characteristics

\subsection{Parameter Analysis Of Resistance Band Antenna}

In the circular slot of the UWB antenna, a cross type open circuit structure is added to form a notch structure unit. Using software to scan different parameters on the cross structure. Due to addition of cross type structure, it will produce a stop band in the band. the scanning of five add parameters, produced a five notch bandwidth in the frequency range of 5.16-7.24GHz.And the maximum bandwidth is add $=1 \mathrm{~mm}$, band-notched width is $1.24 \mathrm{ghz}$ and resistance with center frequency is $6.5 \mathrm{GHz}$, echo gain $-3.22 \mathrm{~dB}$; minimum bandwidth is add $=5 \mathrm{~mm}$, band-notched width is $0.74 \mathrm{GHz}$ and resistance with center frequency for $5.49 \mathrm{GHz}$ echo reflection gain $-1.74 \mathrm{~dB}$. Visible, cross type structure in the high frequency bandwidth, low frequency bandwidth is narrow, S11 gain at high frequency, low frequency and high frequency. It shows that it has a good performance in low frequency band, and the band width can also cover the width of WLAN band. Therefore, the design requirements of the UWB antenna with notch performance can be achieved by using the cross structure[7][8].

After final adjustment, the stop band ultra wide band antenna simulation diagram and parameters are shown in figure (13). 


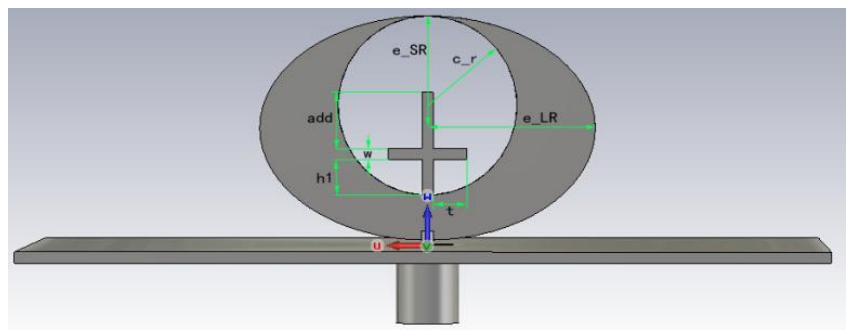

Fig. 13 The model of ultra wide band antenna

In order to further study the performance of the antenna, the radiation characteristics of the antenna in $8 \mathrm{GHz}$ and $4.5 \mathrm{G}$ are studied, and the radiation pattern is obtained, as shown in figure (14) and (15).
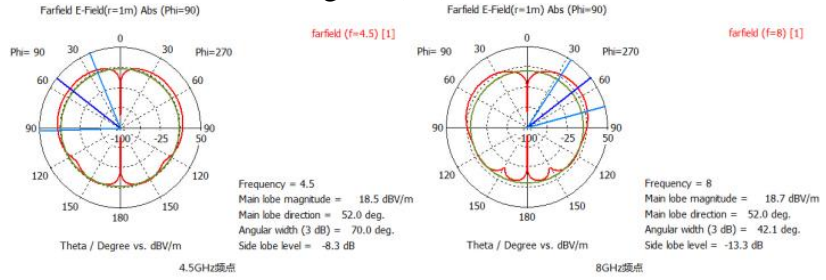

Fig. 14 Ultra wide band antenna E surface direction

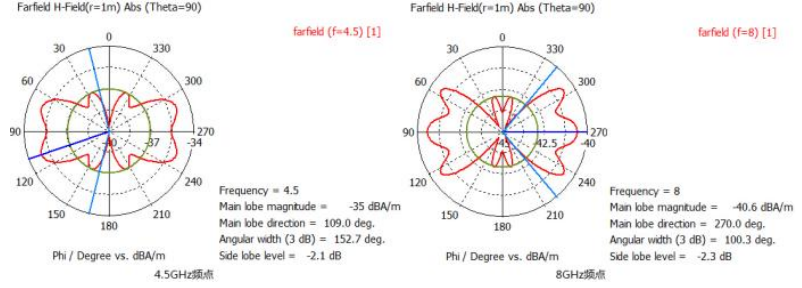

Fig. 15 Ultra wide band antenna $\mathrm{H}$ surface direction

We can be seen from the Fig. 14, 15 shows the radiation pattern of the antenna e surface at the low frequency and high frequency has a nearly omnidirectional radiation characteristics of; $\mathrm{H}$ surface radiation characteristics has certain direction, but with the increase of frequency, $\mathrm{H}$ plane pattern will change, main lobe and concentrated to 90 degrees, side lobe gain becomes lower, indicating that the high frequency more direction, but can still meet the requirements of ultra wide band[9][10].

\section{Conclusions}

This paper first introduce the ultra definition of broadband, the development history and research status of ultra wide band antenna .Expounds the main parameters of the antenna, such as the direction map, direction coefficient, 
voltage VSWR, then introduces the ultra wide band system of antenna and antenna simulation analysis. At the same time, highlight the ultra wide band antenna design technology commonly used in some: including miniaturization technology, realize the trapped wave characteristics of the technology. Finally, based on the theoretical analysis above, two kinds of ultra wide band antennas are designed: monopole ultra wide band antenna, ultra wide band antenna with band notch function. The first monopole ultra wide band antenna at VSWR $<2$, the working band covered by $3.1 \mathrm{GHz}-15 \mathrm{GHz}$. Second kinds of ultra wide band antenna in VSWR<2, the working frequency band is $3.1-5.16 \mathrm{GHz}$ and 5.9 $15 \mathrm{GHz}$; when VSWR $>2$, the antenna band stop band is $5.16-5.9 \mathrm{GHz}$, can be very good to suppress the interference of WLAN system.

\section{References}

1. Ceng Zhaoquan, Liu Jiangnan. An overview and Prospect of ultra wide band technology [J]. Shihezi science and technology, 2012202 (4): 24-26.

2. Jiao Jing. Having depression belt performance of ultra wideband antenna research and design of [D]: [Master thesis. Xi'an: Xi'an Electronic and Science University, 2009.

3. Xu Baolin. Design and application of miniaturized Ultra Wideband Microstrip Antenna with resistance characteristics [D]: [Master's Degree Thesis]. Chengdu: Chengdu University of Technology, 2013.

4. He Yunhong. Research and design of ultra wideband antenna [D]: [Master's Thesis]. Hangzhou: China Jiliang University, 2012.

5. Chen Chuan, Jiao Yongchang, Zhang Li, et al. A kind of ultra wideband antenna with notch characteristics [J]; 2009 National antenna Conference Proceedings (upper) [C]; 2009.

6. Deng Hui. Research and design of ultra wideband antenna with notch characteristics [D]: [Master Dissertation]. Nanchang: East China Jiaotong University, 2013.

7. single Kun. Research and design of time domain miniature ultra wideband antenna [D]: [Master's Degree Thesis] Chengdu: University of Electronic Science and technology of China, 2011.

8. Zhu Chao. Research on Design of ultra wideband antenna and its notch characteristics [D]: [Master's Thesis]. Xi'an: Xi'an Electronic and Science University, 2011.

9. Zhang Jianhua, Hu Wei, Tian Jian. A new type of polygon ultra wideband printed antenna [J]. modern radar.2011,33 (3): 60-62. 
10. Marindra, A.M.J.; Pormwong, S.; Takada, J. Comprehensive characterization of a novel UWB elliptical planar monopole antenna; TENCON 2012 - 2012 IEEE Region 10 Conference. 